\section{Industrielles Recycling - Die Rolle der Industrie für Recycling und moderne Abfallwirtschaft}

Diese Ausgabe der Österreichischen Wasser- und Abfallwirtschaft steht unter dem Motto „Industrielles Recycling“. Ich möchte dies zum Anlass nehmen, die Rolle der Industrie in einem modernen abfallwirtschaftlichen System zu diskutieren. Moderne Abfallwirtschaft zeichnet sich durch den gezielten Einsatz von Kreisläufen aus. Die Kreisläufe des ReUse, des Recyclings und der energetischen Verwertung werden konsequent für wesentliche Abfallströme genutzt. Die Abfallwirtschaft deshalb aber in Kreislaufwirtschaft, Stoffflusswirtschaft oder Ressourcenwirtschaft umzubenennen, ist aus meiner Sicht nicht erforderlich, denn die Aufgabe der „Bewirtschaftung von Abfällen“ bleibt ja weiter bestehen. Aus meiner Sicht sollten wir die Dinge beim richtigen Namen nennen und nicht nur semantisch hinter anderen Begriffen „verstecken“.

Zweifellos hat die österreichische Abfallwirtschaft ein sehr hohes Niveau erreicht (BIPRO 2012). Auch die umfassende Studie „Benchmarking der österreichischen Abfallwirtschaft“ (2015) wies den hohen Erreichungsgrad der abfallwirtschaftlichen Ziele nach. Im Bereich Ressourcennutzung bestehen aber noch Verbesserungspotenziale, die insbesondere in einem optimierten Szenario einer zukünftigen österreichischen Abfallwirtschaft dargestellt wurden. Kern dieses Szenarios sind verstärkte getrennte Sammlung und Recycling.

Recycling findet weitgehend nicht in der klassischen kommunalen oder privaten Abfallwirtschaft statt. Diese Unternehmen und Einrichtungen schaffen vielmehr die Voraussetzungen für ein Recycling durch getrennte Sammlung, Sammel- und Verwertungssysteme, Sortierung und weitgehend mechanische Vorbehandlung. In der Öffentlichkeit wird allerdings z. B. eine Sortieranlage meist als Recyclinganlage angesehen oder verkauft, in ihr werden aber nur Vorprodukte für das nachfolgende Recycling in der Industrie gewonnen. Die klassische Abfallwirtschaft produziert also aus gemischten Abfällen definierte Qualitäten bzw. Rohstoffe für das industrielle Recycling. Diese Zwischen- oder Vorprodukte werden dann in industriellen Prozessen als (Sekundär-)Rohstoff eingesetzt.

Wesentliches Merkmal des Recyclings ist die Substitution eines primären Rohstoffes. So ersetzen sortierte Glasscherben den Primärrohstoff Quarzsand, Altpapier den Rohstoff Holz oder definierter Metallschrott den Rohstoff Erz. Ohne die Glas-, Papier-, Spanplatten-, Kunststoff-, Stahl- und Zementindustrie wäre eine Kreislaufführung von Abfällen nicht denkbar. Glücklicherweise ist Österreich ein Industrieland mit vielfältiger Industrie, die bereits vor Jahrzehnten erkannt hat, dass der Einsatz von Sekundärrohstoffen ökonomische und prozesstechnische Vorteile bringt. Viel zu wenig werden aus meiner Sicht auch die ökologischen Vorteile des industriellen Recyclings dargestellt. Die Vorteile in Bezug auf Energieeffizienz und Treibhausgaseinsparung sind aber signifikant.

Die Industrie ermöglicht der Abfallwirtschaft erst die Kreislaufführung von bestimmten Abfallströmen. Sie hat sich durch diese Anbindung an die Abfallwirtschaft zu einem wesentlichen Teil des abfallwirtschaftlichen Systems entwickelt. Reine Lieferanten-Kunden-Beziehungen sind aus meiner Sicht dabei nicht ausreichend, vielmehr wird die Industrie zukünftig noch mehr Verantwortung für eine hochwertige österreichische Abfallwirtschaft übernehmen und sich zukünftig als wichtiger Teil der Abfallwirtschaft verstehen müssen. Gesetzgebung und Verwaltung sollten diese verstärkte Rolle der Industrie für eine funktionierende Kreislaufwirtschaft anerkennen und fördern. Recycling zu erleichtern und im Vergleich zu alternativen Behandlungen zu bevorzugen, könnte durch gezielte Lenkungsmaßnahmen erreicht werden.

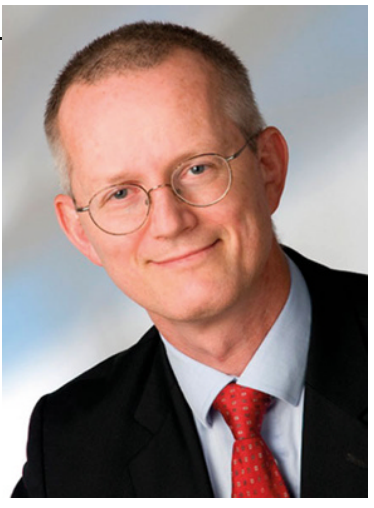

Univ.-Prof. DI Dr. Roland Pomberger 
Ein weiterer Vorteil des Recyclings ist der Aspekt der Ressourcenschonung durch Ersatz von Primärrohstoffen. Dies ist auch ein ausdrückliches Ziel der Abfallwirtschaft (§ 1 AWG 2002). Hier besteht aber auch ein hohes Konfliktpotenzial, denn Sekundäre Rohstoffe substituieren primäre Rohstoffe. Bei internationalen Märkten, z. B. den Metallmärkten, ist dies kein Problem, da international gehandelte Commodities ersetzt werden. Ganz anders ist es aber bei Sekundärrohstoffen, die in lokalen Märkten primäre Rohstoffe verdrängen. Es ist offensichtlich, dass z. B. Recyclingbaustoffe oder Baustoffe aus aufbereiteten Schlacken mit Steinbrüchen und Kiesgruben konkurrieren. Stakeholder der primären Erzeugung versuchen daher auch - mit mehr oder weniger tauglichen Mitteln - die Konkurrenzprodukte zu verhindern. Diese Auseinandersetzung wird tw. auch mit vorgeschobenen Umweltargumenten oder in der Öffentlichkeit ausgetragen. Wir müssen uns bei Verstärkung des Recyclings darauf gefasst machen, dass sich diese Auseinandersetzungen zwischen primärer und sekundärer Rohstoffwirtschaft weiter verschärfen werden. Der Gesetzgeber sollte sich davon aber nicht beeindrucken lassen und Recycling durch geeignete Regelungen, auch Lenkungsmaßnahmen, fördern und verstärken.

In dieser Ausgabe werden mehrere industrielle Recyclinglösungen vorgestellt. Sie umfassen ein weites Spektrum und reichen von kritischen Rohstoffen über rohstoffliches Recycling von Kunststoffabfällen bis zu den Möglichkeiten der Automobilindustrie. Konkrete Forschungsprojekte der Universitäten mit der Industrie und der Abfallwirtschaft können dazu beitragen, dass in Zukunft noch mehr Stoffströme als Rohstoffe genutzt werden und die abfallwirtschaftlichen Ziele noch besser erreicht werden.

\section{Univ.-Prof. DI Dr. R. Pomberger (四)}

Lehrstuhl für Abfallverwertungstechnik und Abfallwirtschaft, Department für Umwelt- und Energieverfahrenstechnik, Montanuniversität Leoben,

Franz-Josef-Straße 18,

8700 Leoben, Österreich

roland.pomberger@unileoben.ac.at 